\title{
Estrutura fatorial do WISC-III em crianças com dificuldades de aprendizagem
}

\author{
Francisco Antonio Soto Vidal - Universidade Católica de Pelotas, Rio Grande do Sul, Brasil \\ Vera Lúcia Marques de Figueiredo - Universidade Católica de Pelotas, Rio Grande do Sul, Brasil
}

\begin{abstract}
Resumo
O presente trabalho busca verificar qual o modelo fatorial mais adequado para o WISC-III no grupo clínico das crianças com dificuldades de aprendizagem. Foram analisados 263 protocolos de testes aplicados em alunos de escolas públicas, encaminhados por seus professores para avaliação psicológica. Foram utilizadas as técnicas estatísticas da análise fatorial exploratória e confirmatória. O presente estudo, além de corroborar a estrutura fatorial definida na padronização brasileira, vai ao encontro dos resultados da pesquisa internacional quanto à definição do modelo de quatro fatores como o de melhor ajuste para esse grupo clínico. Apesar de também ter identificado dois modelos trifatoriais como vantajosos quanto ao ajuste, parcimônia e interpretabilidade teórica, a estrutura quadrifatorial é a mais indicada para interpretar clinicamente as pontuações que expressam as habilidades cognitivas do grupo estudado, uma vez que permite aproveitar as normas existentes do WISC-III para a população geral.

Palavras-chave: Escalas de Wechsler, Análise fatorial, Validade, Dificuldades escolares.
\end{abstract}

\section{WISC-III factor structure for children with learning disabilities}

\begin{abstract}
This work contributes to the investigation of a factorial model that is more appropriate for Brazilian children with learning disabilities (LD). 263 WISC-III test protocols of public school students with academic difficulties referred by their teachers for psychological evaluation were analyzed. Statistical techniques of exploratory factor analysis and confirmatory factor analysis were performed. This study, besides corroborating the factor structure defined in the Brazilian standardization, meets the results of the international research for the definition of four-factor model as the best adjusting for the LD population. Although we have also identified two three-factor models as advantageous as to the fit, parsimony and theoretical interpretability, the four-factor structure is the most suitable for clinical interpretation of the scores that express the LD group cognitive abilities, since it allows us to leverage existing standards of WISC-III for the general population.

Keywords: Wechsler scales, Factor analysis, Validity, School difficulties.
\end{abstract}

Estructura factorial del WISC-III en niños con problemas de aprendizaje

\begin{abstract}
Resumen
El presente trabajo busca verificar cual el modelo factorial más adecuado para el WISC-III en el grupo clínico de niños con dificultades de aprendizaje. Se analizaron 263 testes aplicados en alumnos de escuelas públicas, encaminados por sus maestros para evaluación psicológica. Se utilizaron las técnicas estadísticas del análisis factorial exploratorio y confirmatorio. Este estudio, además de corroborar la estructura factorial definida en la estandarización brasileña, coincide con los resultados de la investigación internacional en cuanto a la definición del modelo de cuatro factores como de mejor ajuste para ese grupo clínico. Además de haber también identificado dos modelos trifactoriales como ventajosos en cuanto al ajuste, a la economía factorial y a la interpretabilidad teórica, la estructura de cuatro factores es la más indicada para interpretar clinicamente los puntajes que expresan las habilidades cognitivas del grupo estudiado, pues permite aprovechar las normas ya existentes del WISC-III para la población general.

Palabras clave: Escala de inteligencia de wechsler para niños, Análisis factorial, Dificultades de aprendizaje.
\end{abstract}

Uma das formas de se verificar a validade de um instrumento psicológico é buscando evidências à base de sua estrutura interna, seja pesquisando as relações entre o teste e seus itens, seja verificando o agrupamento dos itens em fatores predefinidos (Alves, Souza \& Baptista, 2011). Sempre que um instrumento de avaliação for aplicado em novos grupos populacionais, deve passar por adaptações e pela revisão de validade de sua estrutura (Reichenheim \& Moraes, 2007).

O WISC-III (Wechsler Intelligence Scale for Children Third Edition) avalia a inteligência geral em crianças e adolescentes, além de um conglomerado de habilidades específicas (Wechsler, 1991). O teste calcula três quocientes intelectuais - QI verbal (QIV), QI de execução (QIE), QI total (QIT) - e quatro índices Disponivel em www.scielo.br fatoriais: compreensão verbal (CV), resistência à distração (RD), organização perceptual (OP) e velocidade de processamento (VP). Mediante análises qualitativas, é usado para diagnosticar dificuldades na leitura e na escrita (Cavalini, 2008). O instrumento foi traduzido e adaptado para a população brasileira (Figueiredo, 2001) sem investigar sua validação para o grupo clínico das dificuldades de aprendizagem (DA) foco abordado pelo presente estudo.

$O$ estudo dos modelos fatoriais das escalas Wechsler começou a desenvolver-se ao surgir, em 1974, o WISC-R, a versão anterior ao WISC-III. As pesquisas logo encontraram apoio para o modelo de dois fatores, tanto para a população geral quanto para diversos grupos especiais (Anderson \& Dixon, 1995; Donders, 1993). Começou a ser investigado um 
terceiro fator, identificado por Kaufman em 1979 e denominado Freedom from Distractibility (Blaha \& Wallbrown, 1996) ou resistência à distração (RD).

O WISC-III criou um subteste (Procurar Símbolos) e propôs o quarto fator, VP. A maioria das pesquisas ratificou, para a população geral, esse novo modelo (Kamphaus, Benson, Hutchinson \& Platt, 1994; Keith \& Witta, 1997; Roid, Prifitera \& Weiss, 1993). A estrutura de quatro fatores também foi corroborada para grupos clínicos (Donders \& Warschausky, 1996; Maller \& Braden, 1993; Sullivan \& Montoya, 1997; Tiholov, Zawallich \& Janzen, 1996; Tupa, Wright \& Fristad, 1997).

A padronização brasileira do WISC-III informou sobre a estrutura fatorial do teste, encontrando o modelo de quatro fatores como o mais adequado. Outro modelo trifatorial - com os seis subtestes verbais, os quatro de OP e os dois de VP - ficou em segundo lugar quanto ao ajuste aos dados (Figueiredo, 2001).

\section{Modelos fatoriais do WISC-III para dificuldades de aprendizagem}

A dificuldade de aprendizagem (DA) é um distúrbio cognitivo que afeta a aprendizagem, tanto nas habilidades especificamente escolares - a leitura, o soletrar e a aritmética (OMS, 1993) - quanto nas áreas da fala, das emoções, do comportamento ou da concentração da atenção. Tais alterações podem ocorrer em consequência de características do indivíduo, problemas do sistema educacional ou causas ambientais (Golbert \& Moojen, 2000; Paín, 1992).

A partir dos anos 1970, diversos estudos com o WISC-R já apontavam a relativa debilidade do fator RD em amostras com DA (Petersen \& Hart, 1979; Schooler, Beebe \& Koepke, 1978; Snow, Cohen \& Holliman, 1985; Zarske, Moore \& Petersen, 1981). Entretanto, um estudo norte-americano (Fischer \& Dean, 1987) e um holandês (Meesters, van Gastel, Ghys \& Merckelbach, 1998) corroboraram os três fatores do teste (QIV, QIE e RD) em crianças com DA.

Depois de publicado o WISC-III, dois estudos fatoriais pesquisaram o grupo clínico DA: uma análise fatorial confirmatória numa amostra mista $(\mathrm{n}=167)$ com deficiências em leitura e atenção (Wechsler, 1991) e uma análise fatorial exploratória com 78 alunos de uma escola para transtornos de aprendizagem (Hishinuma \& Yamakawa, 1993) encontraram apoio para os quatro fatores, apesar de não utilizarem as mesmas técnicas nem o mesmo critério de seleção e tamanhos amostrais.

Posteriormente, outros três estudos utilizando análise fatorial exploratória em amostras com DA questionaram a validade do modelo quadrifatorial, contradizendo os achados anteriores para a população geral e para grupos especiais. Logerquist-Hansen e Barona (1994) verificaram uma estrutura de três fatores: CV com seis itens, OP sem Arranjo de Figuras e VP com seus subtestes, sem apoio para RD. Em outra amostra ( $n=121)$, Kush e Watkins (1994) encontraram evidências para os fatores $\mathrm{CV}$ (com aritmética), OP (sem arranjo) e VP, mas sem apoio claro para a validade de RD. Em 1996, Kush encontrou apoio à validade do fator CV (com aritmética) e OP (com os quatro subtestes), sem constatar grande força de VP, enquanto RD não obteve evidência de validade.

Nos anos seguintes, novas pesquisas apoiaram o modelo bifatorial, remarcando as dúvidas sobre RD. Com 11 subtestes (sem procurar símbolos), Slate e Jones (1995) encontraram, mediante análise fatorial exploratória, evidência preliminar para dois grandes fatores, sem resultados conclusivos, provavelmente pela reduzida amostra $(\mathrm{n}=58)$. Poulson (1995: citado por Watkins \& Kush, 2002) realizou uma análise fatorial confirmatória com 200 alunos, achando o melhor ajuste e parcimônia para um modelo com CV e OP.

Outras pesquisas utilizando análise fatorial confirmatória apoiaram o modelo de quatro fatores. Estudando crianças e jovens com dificuldades (DA e retardo mental, $n=715)$, Konold, Kush e Canivez (1997) compararam cinco modelos, concluindo que a solução quadrifatorial era a melhor para esse grupo. Em 1999, Grice, Krohn e Logerquist testaram seis modelos para os resultados de duas amostras com DA ( $\mathrm{n}=280 ; \mathrm{n}=240$ ). Um modelo de quatro fatores explicou os dados de forma mais acurada e estável; porém, um modelo trifatorial sem procurar símbolos também teve bom ajuste e fidedignidade, denotando a dificuldade para diferenciar os dois modelos.

Entretanto, uma meta-análise totalizando 934 protocolos de seis estudos fatoriais com o WISC-III encontrou como melhor solução um modelo trifatorial configurado com a subescala verbal e os fatores OP e VP (Ravert \& Watkins, 2000: citado por Watkins \& Kush, 2002). Essa mesma estrutura - que exclui RD mas não seus dois subtestes - foi bem avaliada na padronização brasileira (Figueiredo, 2001).

Outra pesquisa com análise fatorial confirmatória (Burton e cols., 2001) deu apoio a um modelo de cinco fatores, usando escores de 318 crianças com dificuldades diversas. Avaliados nove modelos, identificaram-se os fatores: $\mathrm{CV}, \mathrm{RD}$, práxis construtiva (OP, sem Arranjo de Figuras), VP e raciocínio visual (fator novo, formado por arranjo de figuras). Labirintos não foi incluído por não melhorar o ajuste. 
Indiretamente, a estrutura desse modelo apoiava os quatro fatores de Wechsler.

Hale, Fiorello, Kavanagh, Hoeppner e Gaither (2001) encontraram, para 174 crianças com DA, que os fatores tradicionais de primeira ordem do WISC-III explicavam grande parte da variância do rendimento escolar, conclusão que reforçou a utilidade dos quatro fatores nesse grupo. Outro estudo (Kush e cols., 2001) examinou, mediante análise fatorial exploratória e confirmatória os resultados de 348 afro-descendentes encaminhados para avaliação psicológica (59\% deles com DA), achando evidência de um fator principal, assim como dos esperados componentes verbal e de execução.

No maior estudo encontrado nesta revisão, Watkins e Kush (2002) aplicaram análise fatorial confirmatória a 12 modelos teóricos para alunos com DA $(n=1201)$. Quatro modelos foram tidos como plausíveis: o de Wechsler, o modelo hierárquico quadrifatorial de primeira ordem, um modelo bifatorial com quatro fatores de primeira ordem e um modelo bifatorial com três fatores de primeira ordem. Esses resultados trouxeram evidências da robustez dos fatores CV, OP e VP, e uma fraca validade de RD.

$\mathrm{Na}$ Austrália, uma análise fatorial confirmatória com 579 crianças encaminhadas a educação especial por dificuldades escolares testou modelos de um, dois, três e quatro fatores e mostrou que o modelo quadrifatorial se ajustava melhor aos dados (Cockshott, Marsh \& Hine, 2006).

A literatura não mostra com clareza qual o melhor modelo fatorial para a população DA: sete dos 15 estudos revisados preferiram o modelo quadrifatorial, quatro apoiaram uma solução trifatorial sem RD, três destacaram os dois fatores clássicos do WISC e um optou por um modelo menos comum, de cinco fatores. A falta de congruência nesse quadro pode dever-se à diversidade de técnicas, a diferenças educacionais, a variados tamanhos amostrais (entre 58 e 1.201) e a diversos vieses, especialmente o de seleção. Contudo, podemos extrair algumas conclusões gerais.

Segundo a presente revisão, o fator g não está no foco das pesquisas nem aparece questionado; antes, ele é avaliado favoravelmente por três fatorações exploratórias. A verdadeira polêmica parece apontar ao fator RD, cuja validade conceitual é posta em dúvida pela metade das pesquisas. É preciso notar que o questionamento de $\mathrm{RD}$ como fator (não necessariamente dos dois subtestes que o constituem) se refere à definição de seu conteúdo teórico, sem afetar seu uso na investigação ou na interpretação dos perfis clínicos. Tampouco parece relacionar-se com alguma solução fatorial, haja vista que os modelos pesquisados - sejam de dois sejam de mais fatores - coincidem em apontar a robustez de três fatores: CV e OP têm sido investigados desde a primeira versão do teste e seguem vigentes para a população com DA, enquanto VP, que na presente revisão foi questionado por somente um estudo, permanece o mesmo, persistentemente, em modelos de três, quatro e cinco fatores. Quanto à diversidade metodológica, o questionamento de RD provém das fatorações exploratórias, onde melhor se observa a debilidade desse fator; contrariamente, as técnicas confirmatórias abonam seu uso, mesmo sem considerá-lo o mais robusto.

É de se notar também que apenas uma entre sete análises exploratórias em amostras com DA assinale o modelo quadrifatorial como aceitável, enquanto os estudos de tipo confirmatório tendem a assegurar a vigência da estrutura quadrifatorial. Essa diferença pode dever-se aos objetivos dos dois métodos, que serão descritos na próxima seção.

Buscando definir o modelo fatorial mais adequado para crianças brasileiras com dificuldades de aprendizagem, o presente estudo enfatizou as contribuições das análises de tipo confirmatório. Ao mesmo tempo, esta pesquisa contribui ao estudo da validade do WISC-III para grupos clínicos no contexto brasileiro.

\section{Método}

\section{População-alvo}

Alunos de escolas públicas de duas cidades do Sul do Brasil, com idades entre 6 e 16 anos, apresentando queixas de DA.

\section{Participantes}

Amostra de conveniência, constituída por 263 crianças e adolescentes avaliados mediante o WISC-III em um estudo anterior (Figueiredo, Quevedo, Gomes \& Pappen, 2007). Os participantes foram inicialmente encaminhados por seus professores para avaliação psicológica por apresentarem problemas na aprendizagem, de origens diversas (psicológicas, sociais ou neurológicas). Excluíram-se alunos com mais de três repetências na mesma série ou com evidentes deficiências visuais, auditivas e/ou motoras. De acordo com as autoras do estudo, a amostra apresentou predominância do sexo masculino (69\%), média de idade de 9 anos $(\mathrm{DP}=2)$ e maioria proveniente de escolas estaduais $(80 \%)$.

\section{Procedimento}

Os dados foram captados por um período de três anos junto aos serviços psicológicos das escolas que aceitaram participar do estudo. Ainda conforme o 
estudo referido no parágrafo acima, o WISC-III foi administrado na própria escola do aluno, após a assinatura do termo de consentimento informado pelos responsáveis. A aplicação realizou-se em sessão única, por bolsistas de iniciação científica treinados pelas pesquisadoras. O projeto foi aprovado pelo Comitê de Ética em Pesquisa da Universidade Federal do Rio Grande do Sul (reunião no 26, ata $n^{\circ} 47$ ).

\section{Procedimentos da análise de dados}

Uma análise exploratória dos dados realizada pelo presente estudo verificou, para cada variável (escores ponderados nos 12 subtestes e os sete escores em QIs): três estatísticas descritivas (média, desvio padrão e valores extremos) e a possível existência de casos omissos e de valores atípicos (outliers). Foram pesquisadas também características de configuração das distribuições, como homoscedasticidade, simetria e curtose. Nesses procedimentos, usou-se o pacote estatístico SPSS 13.0 for Windows.

\section{Análise fatorial}

A análise fatorial (AF) é uma técnica estatística multivariada que, mediante a análise de correlações, mostra quantos e quais fatores subjazem a um número de variáveis. $\mathrm{O}$ modelo da AF se baseia nos princípios da causalidade dos fatores e da parcimônia ou economia do modelo. Pelo primeiro, os fatores podem explicar uma parte da covariância entre as variáveis observadas; pelo segundo, a AF procura o menor número de fatores que expliquem a maior porcentagem possível de covariância (Pasquali, 2005). Neste estudo foram utilizadas técnicas de AF exploratória (AFE) e confirmatória (AFC).

$\mathrm{Na}$ AFE, os fatores são buscados mediante a livre observação das correlações, antes de se definir uma solução específica, o que permite gerar hipóteses explicativas sobre os fatores do comportamento (Tabachnick \& Fidell, 2001). A AFC impõe à matriz das correlações, como postulado teórico, uma estrutura fatorial determinada e "verifica, em seguida, se esta se adapta aos dados empíricos expressos pela mesma matriz" (Pasquali, 2005, p. 113). Seu fim é provar qual de vários modelos teóricos melhor se ajusta aos dados amostrais (Kush \& cols., 2001). Gerbing e Hamilton (1996: citados por Kush \& cols., 2001) mostraram que a AFE ajuda o pesquisador a recuperar um modelo de medida subjacente, que logo pode ser avaliado pela AFC, e Carroll (1995: citado por Kush \& cols., 2001) chega a recomendar que as duas formas sejam efetuadas e relatadas, no estudo de habilidades cognitivas.

\section{Análise fatorial exploratória}

$\mathrm{Na}$ extração de fatores, que utilizou o pacote estatístico SPSS 13.0 for Windows e os procedimentos indicados por Pasquali (2005), foram usadas duas técnicas: a análise dos componentes principais (ACP) e a fatoração dos eixos principais (FEP). A primeira serve basicamente para estimar o número mínimo de componentes que explicariam suficientemente a variância dos dados, enquanto a FEP é um método de maior precisão e mais adequado para testar hipóteses teóricas, que permite confirmar essa estimativa inicial da ACP. Para a análise em mais de um fator, usou-se neste estudo a rotação fatorial oblíqua, tendo em vista a alta intercorrelação dos fatores no WISC-III (Figueiredo, 2001; Kush, 1996).

\section{Análise fatorial confirmatória}

No atual estudo, foram testados cinco modelos não-hierárquicos: o modelo de um fator geral com 12 subtestes (M1), um modelo bifatorial com os 5 subtestes padrões do QIV e os 5 do QIE (M2), um modelo de três fatores com os 6 subtestes verbais, os 4 do fator OP e os 2 de VP (M3a), um segundo modelo trifatorial com os 4 subtestes do fator $\mathrm{CV}$, os 2 de RD e os 6 de Execução (M3b), e o modelo tradicional de quatro fatores (M4). Para o teste de ajuste dos modelos, foram analisados os seguintes índices: razão $\chi^{2} /$ g.l. (qui-quadrado por graus de liberdade), os índices de qualidade de ajuste CFI (comparative fit index), GFI (goodness-of-fit index) e AGFI (adjusted goodness-offit index) e o índice residual RMSEA (root mean square error of approximation). Todos os procedimentos da AFC foram realizados mediante o pacote estatístico AMOS 17.0.

\section{Resultados}

\section{Análise exploratória de dados}

A estatística descritiva encontrou médias mais baixas que as da padronização, como era esperável neste grupo clínico. A distribuição por níveis de QI, por exemplo, mostrou cerca de $4 \%$ de participantes com inteligência superior à média, $22 \%$ no nível intelectual médio, $43 \%$ abaixo da média intelectual sem deficiência, e $30 \%$ de casos com deficiência mental. Outras medidas descritivas, como os valores de assimetria e curtose, apontaram uma acentuada dispersão dos dados.

\section{Análise fatorial exploratória}

Nos procedimentos prévios da AFE, as altas comunalidades iniciais entre as variáveis e correlações superiores a 0,30 sugeriram a fatorabilidade da matriz. $\mathrm{O}$ determinante $(0,001)$ e o índice $\mathrm{KMO}(0,94)$ 
confirmaram que o número de variáveis poderia ser reduzido. Pelo método de inspeção visual do gráfico de declive (scree plot), confirmou-se a existência clara de um grande fator (autovalor $=6,75$ ) e até mesmo outros três componentes mais fracos, com autovalores superiores a 0,70 (Joliffe, 1972: citado por Pasquali, 2005). Quatro fatores explicariam $76 \%$ da variância. Para um fator, o método da FEP revelou cargas fatoriais entre $0,80 \mathrm{e}$ 0,60. A ACP distinguiu com clareza dois grupos de seis subtestes - os verbais e os de execução -, mas a FEP identificou somente um fator, mostrando a pouca força do modelo bifatorial nessa amostra. $\mathrm{Na}$ busca de três fatores, a FEP claramente identificou F1 como o conjunto verbal, F2 como os subtestes de OP, e F3 como os de VP. A mesma técnica não sustentou o modelo de quatro fatores. Portanto, três modelos foram, nessa fase, os mais viáveis para o grupo DA: o unifatorial, o bifatorial e o trifatorial, estruturado este último com um fator verbal e os fatores $\mathrm{OP}$ e VP. Todos eles foram revisados pela análise confirmatória, foco principal deste estudo. Os resultados são apresentados mais detalhadamente em Vidal (2010).

\section{Análise fatorial confirmatória}

Os cinco modelos testados mostraram medidas satisfatórias de ajuste aos dados (v. Tabela 1). Dentre eles, M4 constituiu-se como o melhor modelo para esta amostra, com a mais baixa razão $\chi^{2} /$ g.l., o mais baixo RMSEA e os mais altos índices GFI, AGFI e CFI, sem necessidade de especificações posteriores.

Tabela 1. Índices de ajuste na análise fatorial confirmatória

\begin{tabular}{cccccc}
\hline Índices & M1 & M2 & M3a & M3b & M4 \\
\hline$\chi^{2}$ & 73,6 & 72,2 & 101,8 & 86,6 & 79,5 \\
g.l. & 31 & 34 & 51 & 50 & 48 \\
P & 0,000 & 0,000 & 0,000 & 0,003 & 0,003 \\
$\chi^{2} /$ g.l. & 2,4 & 2,1 & 2,0 & 1,7 & 1,7 \\
RMSEA & 0,072 & 0,065 & 0,062 & 0,053 & 0,050 \\
GFI & 0,947 & 0,951 & 0,94 & 0,952 & 0,954 \\
AGFI & 0,905 & 0,92 & 0,908 & 0,924 & 0,926 \\
CFI & 0,972 & 0,975 & 0,972 & 0,980 & 0,982 \\
\hline
\end{tabular}

Legenda: $\chi^{2}$ /g.l razão qui-quadrado por graus de liberdade; P probabilidade; RMSEA root mean square error of approximation; GFI goodness-of-fit index; AGFI adjusted goodness-of-fit index; CFI comparative fit index

A Figura 1 mostra a representação gráfica do modelo, que consta dos indicadores ou variáveis observáveis em retângulos (os 12 subtestes), os erros padrão de cada variável (de e1 a e12), os fatores em elipses (os quatro índices fatoriais), as setas unidirecionais - que assinalam as influências sobre as
12 variáveis medidas - e as seis correlações entre os fatores, indicadas por setas curvas bidirecionais. Acima das setas do gráfico, aparece cada uma das cargas fatoriais padronizadas resultantes da AFC (oscilando entre 0,69 e 0,84$)$ e as correlações entre os fatores (entre 0,90 e 0,74 ). 


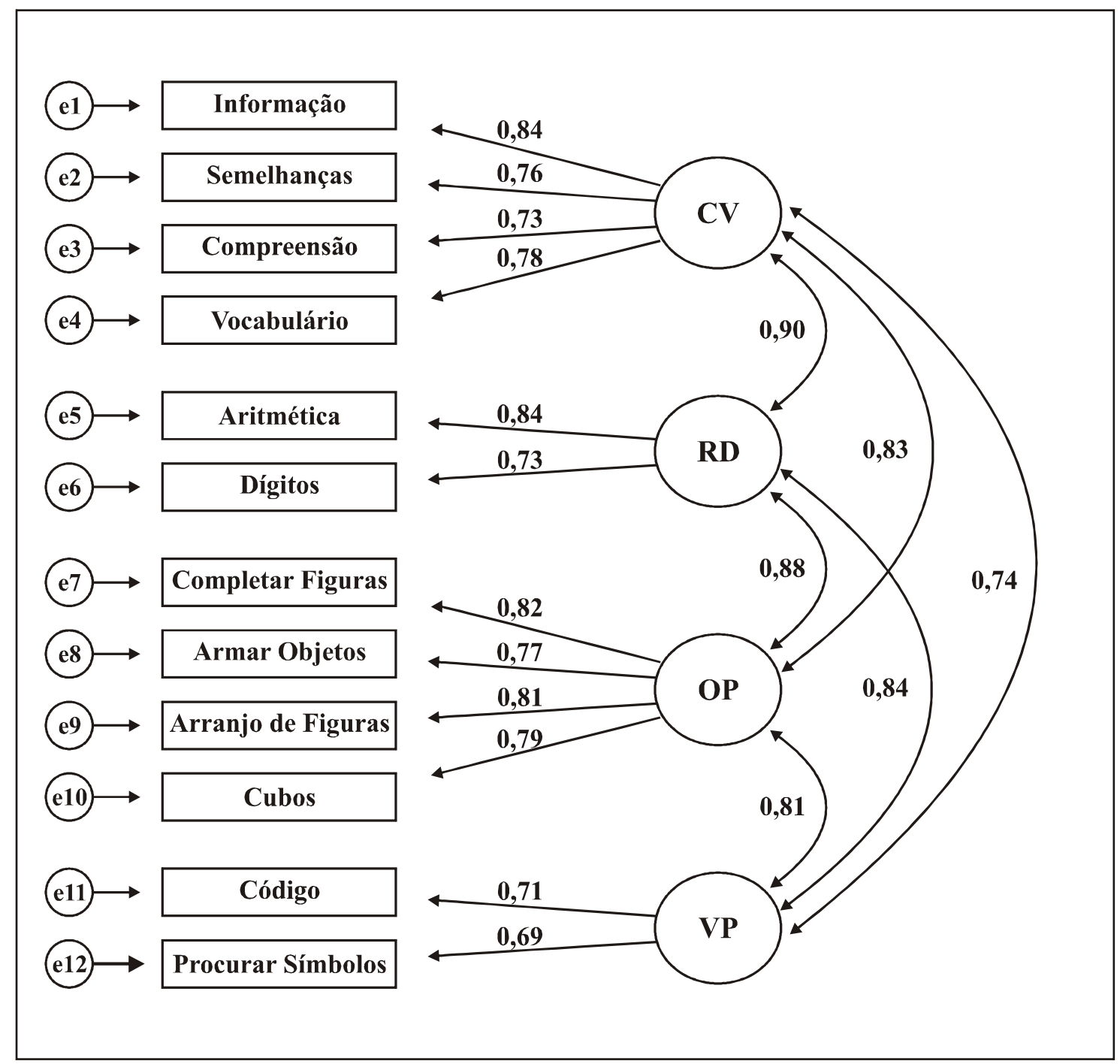

Figura 1. Modelo Quadrifatorial M4

Legenda: CV compreensão verbal; RD resistência à distração; OP organização perceptual; VP velocidade de processamento; e1 erro-padrão 1; e2 erro-padrão 2 etc.

M3b teve igualmente um bom ajuste, com praticamente as mesmas medidas de M4, obtidas ao acrescentar-se uma especificação: a correlação entre o erro-padrão de código e o de procurar símbolos (subtestes do mesmo fator). O ajuste de M3a teve avaliação semelhante, situando-se em terceiro lugar de qualidade, dentro dos índices de ajuste aceitáveis (razão $\chi^{2} /$ g.l.=2). M2 tampouco requereu reespecificações, com todos os outros índices em nível satisfatório. Por último, o modelo unifatorial com 10 subtestes apresentou razão $\chi^{2} /$ g.l. um pouco mais alta e necessitou reespecificar quatro parâmetros para situarse no nível de ajuste aceitável: correlações entre o erro padrão de informação e o de vocabulário, o erro padrão de completar figuras e armar objetos, o erro padrão de armar objetos e arranjo de figuras, e o erro padrão de cubos e armar objetos - todas elas com pertinência teórica, por serem ligações dentro do mesmo fator.

\section{Discussão}

A estatística descritiva mostrou a amplitude da amostra segundo a capacidade geral (QIT), desde a deficiência até inteligência superior, refletindo a heterogênea definição de DA e a variedade de causas que podem originar as dificuldades. Entre os QIs mais baixos, deve-se notar que alguns deles podem ser "deficiências funcionais", ou seja, não decorrem de uma estrutura realmente deficitária mas de uma interação inadequada com o meio, por razões como: carências socioculturais, problemas pedagógicos, ou alterações emocionais e do comportamento que afetam o desempenho intelectual. Por outro lado, esse amplo espectro pode conter também muitas dificuldades reais de aprendizagem que se atribuem falsamente a disfunções neurológicas.

Psico-USF, Bragança Paulista, v. 18, n. 1, p. 23-32, jan./abril 2013 
As baixas médias obtidas nos subtestes podem vincular-se ao fator socioeconômico, presente na amostra aqui estudada, toda ela proveniente de escolas públicas. Nos Estados Unidos, a questão cruza com o fator étnico e cultural, que algumas pesquisas já observaram influir negativamente nos resultados do WISC-III (Kush \& Watkins, 1994; Kush \& Watkins, 2007; Kush e cols., 2001; Watkins \& Kush, 2002).

As análises fatoriais exploratórias mostraram evidências de robustez para CV, OP e VP, constituídos pelos subtestes esperados. RD não ficou bem definido nessa fase, denotando alguma falha teórica na sua definição como construto, como a literatura já previa. Nessas fatorações, por outro lado, os subtestes de RD - Aritmética e Dígitos - carregaram sempre como os menos fortes da área verbal, sugerindo a existência de um fator diferenciado. Por outro lado, a AFC (v. Figura 1) mostrou cargas altas dos dois subtestes de RD.

As explorações fatoriais evidenciaram também a capacidade do WISC-III para estimar a inteligência geral no grupo DA, o que vai ao encontro de estudos que observaram a força do fator $g$ na população brasileira (Figueiredo, 2001) e para o grupo DA nos Estados Unidos (Kush \& Watkins, 1994; Kush e cols., 2001). Entretanto, a atual AFC situou o modelo unifatorial entre os modelos aceitáveis, fato que, mesmo com a necessidade de algumas reespecificações do modelo, justifica o uso de uma pontuação de QI total na interpretação dos resultados do teste.

O modelo bifatorial teve evidência confirmatória de bom ajuste aos dados, apesar de as fatorações exploratórias não terem separado claramente o construto verbal e o de execução, provavelmente pela forte presença do fator $g$. Esses resultados apoiam a literatura sobre o WISC-III, que, sem anular a importância dos dois fatores, os integra ao modelo quadrifatorial sob uma estrutura hierárquica, em que verbal e execução contêm, cada um, dois fatores de ordem inferior.

No presente estudo, os dois modelos de três fatores (M3a e M3b) mostraram bom ajuste aos dados, evidenciando que todos os subtestes são relevantes na avaliação de crianças com DA e confirmando que os quatro fatores têm uma consistência mútua. Ambos os modelos apresentaram ainda, em relação ao quadrifatorial, a importante vantagem da parcimônia. Um deles - o que agrupava a área verbal num só fator, distinguindo VP - apresentou concordância com a principal conclusão de uma meta-análise realizada nos Estados Unidos (Ravert \& Watkins, 2000: citado por Watkins \& Kush, 2002) e mostrou bom ajuste também na padronização brasileira (Figueiredo, 2001). O segundo modelo trifatorial verificado - que agrupava os subtestes não-verbais, destacando RD - também mostrou bons índices, mas não encontra hoje apoio na literatura, apesar de ter sido inicialmente identificado com os estudos que distinguiam o Terceiro Fator no WISC-R. Com todas suas vantagens, os dois modelos somente poderiam ter aplicação clínica em caso de serem elaboradas normas especiais para sua adequada interpretação.

O modelo quadrifatorial validado pelo WISC-III obteve, segundo a revisão de literatura sobre amostras DA, relativamente poucas evidências positivas em fatorações exploratórias (Hale e cols., 2001; Hishinuma \& Yamakawa, 1993). Isso pode ter-se devido à questionável definição do fator $\mathrm{RD}$, cuja menor robustez foi demonstrada pela técnica exploratória. Entretanto, as pesquisas que utilizaram técnicas confirmatórias - mais adequadas quando se quer comprovar uma teoria já disponível sobre processos latentes, na fase de consolidação do instrumento (Tabachnick \& Fidell, 2001) - mostraram consistentemente que esse modelo segue sendo o mais ajustado (Cockshott, Marsh \& Hine, 2006; Grice, Krohn \& Logerquist, 1999; Konold, Kush \& Canivez, 1997; Watkins \& Kush, 2002). Assim, o presente estudo comprova também, pela análise fatorial confirmatória, a consolidação dos fatores $\mathrm{CV}$, OP, VP e RD para o grupo das dificuldades de aprendizagem.

\section{Considerações finais}

O objetivo deste trabalho foi investigar qual seria o modelo fatorial mais adequado ao grupo clínico das crianças com dificuldades de aprendizagem, buscando confirmar se os construtos descritos para a amostra de padronização brasileira seguiriam sendo válidos para essa população-alvo. Utilizando dois tipos de análise fatorial - exploratória e confirmatória - o estudo corroborou a principal tendência da literatura, que apoia a pertinência da solução quadrifatorial.

Uma das limitações deste estudo foi a utilização de uma amostra de conveniência, compensada por um tamanho amostral significativo. Outras limitações foram: o viés de seleção devido ao foco em escolas públicas, e o caráter local da amostra, que exige precauções ao generalizar os resultados para o âmbito nacional. Para futuros estudos, fica a sugestão de se analisar a estrutura fatorial para diversas faixas etárias intragrupo. Ser o primeiro trabalho nacional que analisa a estrutura fatorial de uma escala Wechsler especificamente num grupo clínico vem a ser o principal mérito do presente estudo.

Buscando definir a estrutura fatorial do WISC-III para a população DA brasileira, este estudo obteve evidências que consolidam a validade interna do teste, 
no que se refere à presença de um fator geral de inteligência (QIT), os dois conjuntos verbal e execução (QIV e QIE) e quatro índices fatoriais (CV, RD, OP e VP). Em apoio à investigação prévia e à proposta definida na padronização, os presentes resultados também constataram a robustez dos fatores $\mathrm{CV}$, OP e VP no grupo clínico DA. Dois modelos trifatoriais mostraram vantagens quanto à parcimônia, o ajuste aos dados e a interpretabilidade, mas o modelo de quatro fatores se mostrou como o de melhores índices nas análises confirmatórias e é, portanto, o mais indicado para aplicação no grupo DA, permitindo aproveitar as normas existentes do WISC-III. A validação desse modelo e dos sete QIs favorece que os psicólogos possam seguir aplicando, ao avaliar crianças com dificuldades de aprendizagem, os mesmos índices fatoriais e os mesmos critérios de interpretação clínica das habilidades cognitivas.

\section{Referências}

Alves, G. A. S., Souza, M. S. \& Baptista, M. N. (2011). Validade e precisão de testes psicológicos. Em R. A. M. Ambiel, I. S. Rabelo, S. V. Pacanaro, G. A. S. Alves \& I. F. A. S. Leme (Orgs.). Avaliação psicológica. São Paulo: Casa do Psicólogo.

Anderson, T. \& Dixon, W. E. (1995). Confirmatory factor analysis of the WISC-R with normal and psychiatric adolescents. Journal of Research on Adolescence, 5(3), 319-332.

Blaha, J. \& Wallbrown, F. H. (1996). Hierarchical factor structure of the Wechsler Intelligence Scale for Children - III. Psychological Assessment, 8(2), 214-218.

Burton, D. B., Sepehri, A., Hecht, F., VandenBroek, A., Ryan, J. J. \& Drabman, R. (2001). A confirmatory factor analysis of the WISC-III in a clinical sample with cross-validation in the standardization sample. Child Neuropsychology, 7(2), 104-116.

Cavalini, S. F. S. (2008). A utilização do WISC-III no diagnóstico das dificuldades de aprendizagem. 123 p. Tese de Doutorado. Instituto de Psicologia, Universidade de São Paulo.

Cockshott, F. C., Marsh, N. V. \& Hine, D. W. (2006). Confirmatory factor analysis of the Wechsler Intelligence Scale for Children - third edition in an Australian clinical sample. Psychological Assessment, 18(3), 353-357.

Donders, J. (1993). Factor structure of the WISC-R in children with traumatic brain injury. Journal of Clinical Psychology, 49(2), 255-260.

Donders, J. \& Warschausky, S. (1996). A structural equation analysis of the WISC-III in children with traumatic head injury. Child Neuropsychology, 2(3), 185-192.

Figueiredo, V. L. M. (2001). Uma adaptação brasileira do Teste De Inteligência WISC-III. 230 p. Tese de Doutorado. Instituto de Psicologia, Universidade de Brasília.

Figueiredo, V. L. M., Quevedo, L., Gomes, G. \& Pappen, L. (2007). Habilidades cognitivas de crianças e adolescentes com distúrbio de aprendizagem. Psico-USF, 12(2), 281-290.

Fischer, W. R. \& Dean, R. S. (1987). A comparative factor analysis of the WISC-R by sex and age with learning disabled children. International Journal of Neuroscience, 35, 217-223.

Golbert, C. S. \& Moojen, S. M. P. (2000). Dificuldades na aprendizagem escolar. Em P. B. Skiennik (Org.). O aluno problema: transtornos emocionais de crianças e adolescentes ( $2^{\mathrm{a}}$ ed.). Porto Alegre: Mercado Aberto.

Grice, J. W., Krohn, E. J. \& Logerquist, S. (1999). Cross-validation of the WISC-III factor structure in two samples of children with learning disabilities. Journal of Psychoeducational Assessment, 17 (3), 236-248.

Hale, J. B., Fiorello, C. A., Kavanagh, J. A., Hoeppner, J. A. B. \& Gaither, R. A. (2001). WISC-III predictors of academic achievement for children with learning disabilities: are global and factor scores comparable? School Psychology Quarterly, 16 (1), 31-55.

Hishinuma, E. S. \& Yamakawa, R. (1993). Construct and criterion-related validity of the WISC-III for exceptional students and those who are "at-risk". Journal of Psychoeducational Assessment: WISC-III Monograph, 11, 94-104.

Kamphaus, R. W., Benson, J., Hutchinson, S. \& Platt, L. O. (1994). Identification of factor models for the WISC-III. Educational \& Psychological Measurement, 54(1), 174-186.

Keith, T. Z. \& Witta, E. L. (1997). Hierarchical and cross-age confirmatory factor analysis of the WISC-III: what does it measure? School Psychology Quarterly, 12(2), 89-107.

Konold, T. R., Kush, J. C., Canivez, G. L. (1997). Factor replication of the WISC-III in three independent samples of children receiving special education. Journal of Psychoeducational Assessment, 15 (2), 123-137.

Kush, J. C. (1996). Factor structure of the WISC-III for students with learning disabilities. Journal of Psychoeducational Assessment, 14(1), 32-40.

Kush, J. C. \& Watkins, M. W. (1994). Factor structure of the WISC-III for Mexican-American, learning disabled students. Relatório de pesquisa. 
Kush, J. C. \& Watkins, M. W. (2007). Structural validity of the WISC-III for a national sample of Native American students. Canadian Journal of School Psychology, 22(2), 235-248.

Kush, J. C., Watkins, M. W., Ward, T. J., Ward, S. B., Canivez, G. L. \& Worrell, F. C. (2001). Construct validity of the WISC-III for white and black students from the WISC-III standardization sample and for black students referred for psychological evaluation. School Psychology Review, 30 (1), 70-88.

Logerquist-Hansen, S. \& Barona, A. (1994). Factor structure of the Wechsler Intelligence Scale for Children-III for Hispanic and Non-Hispanic white cbildren with learning disabilities. Trabalho apresentado na Convenção Anual da American Psychological Association.

Maller, S. \& Braden, J. (1993). The construct and criterion-related validity of the WISC-III with deaf adolescents. Journal of Psychoeducational Assessment: WISC-III Monograph, 11, 105-113.

Meesters, C., van Gastel, N., Ghys, A. \& Merckelbach, H. (1998). Factor analyses of WISC-R and K-ABC in a Dutch sample of children referred for learning disabilities. Journal of Clinical Psychology, 54(8), 10531061.

Organização Mundial da Saúde. (1993). Classificação de Doenças e Problemas Relacionados à Saúde - Décima Revisão. São Paulo: Edusp.

Paín, S. (1992). Diagnóstico e tratamento dos problemas de aprendizagem. Porto Alegre: Artes Médicas.

Pasquali, L. (2005). Análise fatorial para pesquisadores. Brasília: LabPAM.

Petersen, C. R. \& Hart, D. H. (1979). Factor structure of the WISC-R for a clinic-referred population and specific subgroups. Journal of Consulting and Clinical Psychology, 47(3), 643-645.

Reichenheim, M. E. \& Moraes, C. L. (2007). Operacionalização de adaptação transcultural de instrumentos de aferição usados em epidemiologia. Revista de Saúde Pública (online), 41 (4), 665-673.

Roid, G. H., Prifitera, A. \& Weiss, L. G. (1993). Replication of the WISC-III factor structure in an independent sample. Journal of Psychoeducational Assessment: WISC-III Monograph, 11, 6-21.

Schooler, D. L., Beebe, M. S. \& Koepke, T. (1978). Factor analysis of WISC-R scores for children identified as learning disabled, educable mentally impaired, and emotionally impaired. Psychology in the Schools, 15(4), 478-485.

Slate, J. R. \& Jones, C. H. (1995). Preliminary evidence of the validity of the WISC-III for African American students undergoing special education evaluation. Educational and Psychological Measurement, 55(6), 1039-1046.

Snow, J. H., Cohen, M. \& Holliman, W. B. (1985). Learning disability subgroups using clusters analysis of the WISC-R. Journal of Psychoeducational Assessment, 3(4), 391-397.

Sullivan, P. M. \& Montoya, L. A. (1997). Factor analysis of the WISC-III with deaf and hard-ofhearing children. Psychological Assessment, 9(3), 317321.

Tabachnick, B. \& Fidell, L. S. (2001). Using multivariate statistics. San Francisco, CA: Allyn \& Bacon.

Tiholov, T. T., Zawallich, A. \& Janzen, H. L. (1996). Diagnosis based on the WISC-III processing speed factor. Canadian Journal of School Psychology, 12 (1), 23-34.

Tupa, D. J., Wright, M., \& Fristad, M. A. (1997). Confirmatory factor analysis of the WISC-III with child psychiatric inpatients. Psychological Assessment, 9(3), 302-206.

Vidal, F. A. S. (2010). Estrutura fatorial do WISC-III em crianças com dificuldades de aprendizagem: uma validação em amostra brasileira. 54p. Dissertação de Mestrado. Programa de Pós-Graduação em Saúde e Comportamento. Universidade Católica de Pelotas. Pelotas.

Watkins, M. W. \& Kush, J. C. (2002). Confirmatory factor analysis of the WISC-III for students with learning disabilities. Journal of Psychoeducational Assessment, 20(1), 4-19.

Wechsler, D. (1991). Manual for the Wechsler Intelligence Scale for Children-Third edition. San Antonio, TX: The Psychological Corporation.

Zarske, J. A., Moore, C. L. \& Petersen, J. D. (1981). WISC-R factor structures for diagnosed learning disabled Navajo and Papago children. Psychology in the Schools, 18(4), 402-407.
Recebido em: 15/06/2011

Reformulado em: 03/09/2012

Aprovado em: 27/02/2013 
Sobre os autores:

Francisco Antonio Soto Vidal é psicólogo graduado pela Universidad de Chile e mestre em Saúde e Comportamento pela Universidade Católica de Pelotas (UCPel).

Vera Lúcia Marques de Figueiredo é professora do curso de Graduação em Psicologia e do Mestrado em Saúde e Comportamento da Universidade Católica de Pelotas, mestre pela Pontifícia Universidade Católica do Rio Grande do Sul (PUCRS) e doutora pela Universidade de Brasília (UnB).

Contato com os autores:

Universidade Católica de Pelotas - UCPel

Rua Santa Cruz, 1735 - CEP 96015-710 - Pelotas/RS, Brasil.

E-mail: sotovidal@yahoo.com.br 